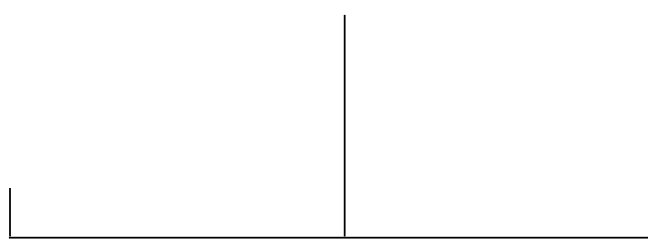

Rev. Latinoam. Psicopat. Fund., São Paulo, v. 15, n. 3, p. 590-608, setembro 2012

\title{
Melancolia e depressão durante o século XIX: uma história conceitual*
}

German E. Berrios

Este artigo analisa historicamente o background ideológico que tornou possível a transformação da noção de melancolia nos conceitos de depressão e transtorno bipolar, a partir das mudanças médicas e psicológicas ocorridas no decorrer do século XIX. A antiga noção de melancolia foi remodelada e sua transição para a doença depressiva foi facilitada pelo conceito de Iipemania de Esquirol, que, pela primeira vez, enfatizou a natureza afetiva primária da doença. Finalmente, uma vez obtidas as condições conceituais necessárias, a melancolia e a mania foram combinadas no conceito de insanidade alternante, periódica, circular, ou de forma dupla, seus rígidos padrões descritivos foram flexibilizados, tendo culminado este processo na sinopse de Kraepelin.

Palavras-chave: Melancolia, transtorno bipolar-história, transtorno depressivo-história, história da psiquiatria

* Publicação original: Melancholia and Depression During the 19th Century: A Conceptual History (Lecture), British Journal of Psychiatry, v. 153, p. 298-304, 1988. Baseado em uma palestra proferida na Reunião de Inverno do Royal College of Psychiatrists, em 29 de outubro de 1987, e dedicado a Dorothy Needham, ilustre historiadora da ciência.

Tradução de Lazslo A. Ávila e RevisãoTécnica de Ana Maria G. Raimundo Oda. 


\begin{tabular}{c|c} 
HISTÓRIA DA \\
PSICOPATOLOGIA, \\
POR GERMAN E. \\
BERRIOS \\
\hline
\end{tabular}

\section{A melancolia antes do século XIX}

O significado de "melancolia" na antiguidade clássica é opaco e tem pouco em comum com seu uso psiquiátrico no século XX (Drabkin, 1955; Heiberg, 1927). Naquele tempo, a melancolia e a mania não eram polos opostos (isto é, não eram definidas como tendo características opostas). A melancolia era definida em termos de características comportamentais evidentes, tais como motilidade reduzida e morosidade (Roccatagliata, 1973; Simon, 1978). Assim, no uso médico, a "melancolia" referia-se a um subtipo de mania e denominava, em geral, os estados de expressão comportamental reduzida. Estes incluiriam distúrbios que podiam "apresentar depressão, agitação, alucinações, estados paranoides e até mesmo demenciais ... o antigo diagnóstico de melancolia não tem nenhuma analogia correta na prática psiquiátrica moderna ..." (Siegel, 1973, p. 274).

A semântica histórica mostra que os sintomas que refletem afeto patológico (tristeza, por exemplo) não faziam parte do conceito, embora, ocasionalmente, uma tal referência poderia ser feita (Berrios, 1985a). Escrevendo sobre esta questão, há mais de 150 anos, Prichard (1835) chegou à mesma conclusão: "Antigamente esta palavra, na linguagem comum, pelo menos, não transmitia uma ideia de tristeza ou abatimento. Melancólico significava simplesmente ser louco" (p. 27).

O significado da melancolia, de fato, só pode ser entendido em termos da teoria humoral (Tracy, 1969). Esta gerava previsões sobre o comportamento do sujeito e sua resposta ao tratamento (Flashar, 1966; Walser, 1968; Starobinski, 1962). Como frequentemente, Jones (1972) tem razão: "a palavra está intimamente ligada tanto com a doutrina dos humores como com a prevalência da malária... no discurso popular, a melancolia e seus cognatos às vezes se aproximam, em significado, ao 'colapso nervoso'. Provavelmente, esse nome foi dado a qualquer condição semelhante à prostração física e mental, produzida pela malária, uma forma da qual (a quartã) se supunha ser causada pela 'bile negra' ('melaina kole')" (p. lviii).

Rev. Latinoam. Psicopat. Fund., São Paulo, v. 15, n. 3, p. 590-608, setembro 2012 
Parece ter havido pouca mudança no significado da melancolia durante a Idade Média (Leibbrand \& Wettley, 1961; Jacquart, 1983), apesar do fato de que, durante este período, foi adotada uma visão mais dura e mais prática da loucura do que até então tinha sido considerada (Kroll \& Bachrach, 1984). Alegou-se, no entanto, que na época de Galeno a noção de melancolia se aproximava das definições atuais (Jackson, 1969, p. 375). O desenvolvimento de conceitos como "nostalgia" (Rosen, 1975; Bégin, 1834; Rauchs, 1985) e "melancolia" ajudou a formar uma família de termos nos quais os sintomas afetivos foram enfatizados (Jackson, 1981, 1983, 1986).

Referências ocasionais a estados que combinam a mania e a melancolia foram feitas antes do século XIX (Briand \& Azemar, 1923), mas em termos das crenças nosográficas mantidas nesses períodos anteriores não faz muito sentido falar de verdadeiras "antecipações" da psicose maníaco-depressiva (Huber, 1985) (para uma história de mania, ver Berrios, 1988a).

\section{O referencial teórico}

A transformação da antiga categoria da melancolia nas suas contrapartes atuais não ocorreu em um vácuo. Mudanças no conceito de doença, na definição psicológica do comportamento e nos princípios taxonômicos foram necessárias. Todas as três mudanças ocorreram no início do século XIX (Berrios, 1988b).

\section{Conceito de doença}

A chamada visão anatomoclínica, desenvolvida no início do século XIX, era de que sinais evidentes de doença poderiam ser correlacionados (sem resíduos) com lesões anatômicas (López Piñero, 1983; Ackerknecht, 1967; Laín Entralgo, 1978). Lesão anatômica, por sua vez, eram definidas em termos de unidades tangíveis de análise. Estas deviam mudar durante o século XIX desde os órgãos para os tecidos e, finalmente, para as células. Fracassos frequentes em identificar tais lesões conduziram, durante a segunda metade do século XIX, à redefinição da "lesão" em termos fisiológicos. Isso tornou possível, inter alia, a incorporação das neuroses à psiquiatria (López Piñero, 1983).

A aplicação da visão anatomoclínica requeria a criação de uma linguagem descritiva (Berrios, 1984). Embora baseada na observação, esta última incluía alguns pressupostos conceituais. O mais importante se refere à legitimidade de 


\begin{tabular}{c|c} 
HISTÓRIA DA \\
PSICOPATOLOGIA, \\
POR GERMAN E. \\
BERIOS \\
\hline
\end{tabular}

separar o comportamento louco em categorias bem definidas. As categorias monolíticas dos 1800 s foram assim progressivamente transformadas, algumas desapareceram, outras foram recuperadas e remodeladas com significado diferente (por exemplo, melancolia), e outras foram deixadas intactas (por exemplo, o delirium) (Berrios, 1981a).

\section{Definição psicológica do comportamento}

Descrições de sintomas foram tornadas possíveis graças à disponibilidade de novas teorias psicológicas (Berrios, 1988b), e o alienista poderia, então, mapear e definir o comportamento e recuperar a experiência subjetiva como outra origem de sintomas (Moreau de Tours, 1859).

Até o final do século XVIII, o associacionismo foi a teoria psicológica predominante na Europa (Warren, 1921; Hoeldtke, 1967). Ela proporcionou o modelo epistemológico para a nova ciência, desde os tempos de Hobbes e Locke. Mas, no final do século XVIII, a psicologia das faculdades mentais experimentou um renascimento, particularmente nas obras de Kant, Reid e Stewart (Berrios, 1988b). A sugestão de que a mente era uma coleção de módulos funcionalmente autônomos forneceu aos alienistas do século XIX um quadro classificatório útil. Por exemplo, Pinel (1809) e Prichard (1835) abandonaram a visão intelectualista da loucura gerada pelo associacionismo lockeano e direcionaram-se para a psicologia das faculdades mentais.

O princípio taxonômico reinante durante o século XIX era a possibilidade de que os três grupos de funções mentais (intelectual, emocional e volitiva) poderiam adoecer separadamente. Assim, as insanidades intelectuais tornaram-se os embriões da esquizofrenia e da paranoia, as insanidades emocionais, da mania e da depressão, e as insanidades volitivas, dos transtornos psicopáticos (Berrios, 1988b).

\section{Princípios taxonômicos}

A filosofia da taxonomia médica também mudou no início do século XIX. Os esquemas botânicos de Lineu, Sauvage, Cullen e outros (Bowman, 1975; Larson, 1971) foram substituídos por princípios empíricos (Desruelles et al, 1934; Baillarger, 1853; Foville, 1872; Vié, 1940). Esses se originavam a partir da comparação dos sintomas e da distribuição frequencial, e até da especulação etiológica. Um novo critério, a história natural da doença (isto é, a dimensão do 
"tempo"), só foi integralmente incorporada à psiquiatria próximo ao fím do século (Del Pistoia, 1971; Lanteri Laura, 1972).

Esforços taxonômicos ao longo do século XIX foram prejudicados pelo sucesso limitado da "visão orgânica" (falha devida, com toda probabilidade, à limitação de recursos tecnológicos) e pela excessiva especulação teórica. Por exemplo, o abuso da explicação genética levou à chamada "teoria da degeneração", que acabou por se tornar um "beco sem saída" (Morel, 1857; Walter, 1956; Wettley, 1959; Friedlander, 1973; Danion et al., 1985; Dowbiggin, 1985).

Uma das consequências interessantes da controvérsia taxonômica foi o desenvolvimento dos pontos de vista unitários e múltiplos da insanidade. Inspirado por um princípio teórico (a indivisibilidade da mente), e pressionado pela exasperação com as classificações da época, um grupo de alienistas apresentou, em meados do século XIX, a visão que haveria apenas uma forma de insanidade e que suas múltiplas apresentações clínicas resultariam de fatores idiossincráticos ou patoplásticos (Llopis, 1954; Menninger et al, 1958; Rennert, 1968; Vliegen, 1980; Beer \& Berrios, 1994*). Foi sugerido, por exemplo, que a mania, a melancolia, a insanidade delirante e a demência vesânica fossem apenas as fases sucessivas da mesma doença. Este é um ponto bastante importante de recordar quando a questão da insanidade circular (transtorno bipolar) é analisada, porque a proposta de que a mania e a melancolia devem ser relacionadas não se baseou apenas na observação clínica (Sedler \& Dessein, 1983), mas também na visão popular naquela época, de que todas as formas de insanidade estariam relacionadas umas às outras.

\section{A melancolia durante o século XIX}

“A melancolia - escreveu John Haslam em 1809 - outra forma em que esta doença (loucura) é suposta existir, segundo o Dr. Ferriar consistiria na 'intensidade da ideia'. Por intensidade da ideia eu presumo que significa que a mente está mais fortemente fixada em, ou mais frequentemente recorre a, um determinado conjunto de ideias, do que quando está em um estado saudável..." (p. 32-33).

Essa percepção era correta. Até o início do século XIX, formas de comportamento que (na nossa perspectiva) tinham pouco em comum, foram incluídas na classe geral da melancolia (Madden, 1966). Não há melhor exemplo

Referência bibliográfica atualizada. (N. da R.)

Rev. Latinoam. Psicopat. Fund., São Paulo, v. 15, n. 3, p. 590-608, setembro 2012 


\begin{tabular}{c|c} 
HISTÓRIA DA \\
PSICOPATOLOGIA, \\
POR GERMAN E. \\
BERRIOS \\
\hline
\end{tabular}

disso do que o conteúdo da Anatomia da melancolia, de Burton (1883). A tristeza pode ocasionalmente ser encontrada entre esses sintomas, mas não era utilizada para a definição, em qualquer sentido real (Lewis, 1934).

Que uma quebra de semântica no significado da melancolia deve ter ocorrido no início do século XIX não é uma sugestão recente. Esquirol (1820) percebeu isso: "a palavra melancolia, consagrada na linguagem popular para descrever o estado habitual de tristeza que afeta alguns indivíduos, deve ser deixada para os poetas e os moralistas cuja solta expressão não está sujeita às restrições da terminologia médica" (p. 148). Prichard (1835) teve opinião semelhante, e Rush (1812), depois de criticar o uso de Cullen, também aconselhou contra o uso da palavra "melancolia" e inventou o novo termo "tristimania".

\section{O conceito da "lipemania"}

Como mencionado acima, a psicologia das faculdades mentais forneceu uma matriz taxonômica para a psiquiatria do século XIX. As doenças eram classificadas, até então, segundo a tradição da nosotaxia more botanico (Lopez Piñero, 1983) em termos de características privilegiadas, tais como a etiologia (por exemplo, Battie, 1758) ou as formas comportamentais (por exemplo, Crichton, 1798; Arnold, 1782). A classificação de melancolia incluía outros sintomas além da perturbação emocional; na verdade, era definida como uma doença de "delírios parciais". Esta origem intelectualista desqualificava a melancolia como o nome certo para as novas "insanidades emocionais".

Assim Esquirol (1820) foi forçado a cunhar a palavra "lipemania" para se referir a "uma doença do cérebro caracterizada por delírios, que são crônicos e fixados sobre temas específicos, ausência de febre e uma tristeza que muitas vezes é debilitante e esmagadora. Não deve ser confundida com a mania que apresenta delírios generalizados e emoções e intelecto excitados, nem com a monomania que apresenta delírios específicos e emoções expansivas e alegres, nem com a demência caracterizada pela incoerência e confusão de ideias resultantes do enfraquecimento..." (p. 151-152, grifos meus).

Esquirol (1820) relatou resultados estatísticos sobre a sua nova doença. As taxas de internação para lipemania foram encontradas aumentadas entre maio e agosto (p. 159), a faixa etária mais acometida era dos 25 aos 45 anos (p. 161), em 110 de 482 casos a "hereditariedade" parecia desempenhar um papel, e suas causas comuns incluíam crise doméstica, luto e relações perturbadas (p. 166). Cerca de um terço de sua coorte morreu, frequentemente de tuberculose. 
Infelizmente, o termo lipemania provou ser, como Delasiauve (1856) afirmou, muito "elástico ... além de ser menos impreciso, não diferindo em termos de conteúdo da antiga palavra melancolia" (p. 382). Delasiauve estava aqui criticando o fato dos delírios "parciais" (um vestígio da antiga noção intelectualista) terem permanecido como um critério de definição da lipemania. Ele sugeriu que o significado de lipemania fosse reduzido ainda mais para se referir a "um exagero e persistência de sentimentos de depressão" (p. 384). Delasiauve passou a atacar Baillarger por considerar a stupidité (estupor) e a lipemania (justamente como veio a ser) como variedades de uma mesma doença (p. 441) (Berrios, 1981b).

O ponto mais alto da história do conceito de lipemania foi atingido na obra de Billod (1856), que tentou uma classificação e um maior refinamento de sua psicopatologia. Billod aceitou que a lipemania fosse definida com base em delírios e afeto tristes, e sugeriu uma classificação quádrupla. Existiria lipemania com delírios tristes e tristeza; delírios tristes e sem tristeza; delírios tristes e transtorno afetivo misto ou alternado (isto incluía os estados bipolares); e ausência de delírios tristes e tristeza. Esta classificação artificial e simétrica permitiu o reconhecimento de cerca de 16 subtipos clínicos. Alguns deles desapareceram desde então (por exemplo lipemania irônica ou religiosa), mas outros (como a lipemania hipocondríaca, a estuporosa e a irritável) ainda são reconhecíveis, embora agora tenham nomes diferentes.

Além da França, o termo lipemania foi utilizado apenas na Espanha. Ele nunca "pegou" na Alemanha, Áustria, Suíça ou Reino Unido, onde a palavra "melancolia" foi mantida. Prichard (1835) não prestou atenção ao termo, nem o fez Griesinger (1867) que, embora citando Esquirol muitas vezes, não tomou conhecimento do seu neologismo. Feuchtersleben (1847) citou o termo uma vez, mas não mencionou sua origem. Bucknill e Tuke (1858) citaram sua origem, mas continuaram usando melancolia com a justificativa de que Esquirol mesmo tinha afirmado que os dois termos poderiam ser usados indistintamente (p. 147). "Depressão mental" gradualmente a substituiu no final do século, e "melancolia" seria cada vez mais usada para nomear um subtipo de depressão psicótica que ocorre em idosos (Chaslin, 1912). A palavra lipemania deve ser considerada como um bom exemplo histórico de como categorias "ponte" raramente sobrevivem. Depois de efetuar a transição de melancolia do conceito intelectualista para o emocional, ela calmamente desapareceu.

\section{O termo "depressão"}

A citação de Delasiauve contém um dos primeiros usos técnicos da palavra "depressão". Em 1860, no entanto, a palavra já era encontrada em dicionários 
HISTÓRIA DA

PSICOPATOLOGIA,

POR GERMAN E.

BERRIOS

médicos "aplicado ao rebaixamento dos espíritos de pessoas que sofrem com a doença" (Mayne, 1860, p. 264). É importante perguntar por que este termo foi necessário. Parece ter sugerido tanto uma "redução" fisiológica quanto metafórica da função emocional e, portanto, tinha a capacidade semântica para nomear fosse uma condição ou um "sintoma". Desta forma, os pontos de vista teóricos anteriores de Griesinger (1867) e as descrições folclóricas da melancolia (como tristeza) poderiam ser reconciliados. Isto é bem ilustrado na primeira (1885) - de muitas - edições do manual de Régis, onde a depressão é definida como "o estado oposto à excitação. Consiste em uma redução da atividade geral que vai desde falhas menores na concentração até a paralisia total..." (p. 77).

Médicos clínicos gerais também parecem ter preferido a depressão à melancolia ou lipemania, talvez porque o termo evocava uma explicação "fisiológica". Sir William Gull (famoso pela anorexia nervosa) a teria usado tão cedo quanto 1868, em seu artigo clássico sobre "hipocondriasis": "sua principal característica é a depressão mental, que ocorre aparentemente sem causa adequada..." (Gull, 1894, p. 287). Até o final do século, "depressão" se tornou sinônimo de melancolia: "uma condição caracterizada por um afundamento dos espíritos, falta de coragem ou iniciativa, e uma tendência a pensamentos sombrios. O sintoma ocorre em condições debilitadas do sistema nervoso, como a neurastenia, e é especialmente característico da melancolia" (Baldwin, 1901, p. 270). Savage (1898) em seu muito popular Insanity and the Allied Neuroses (estudado por muitas gerações de estudantes clínicos do Reino Unido) definiu a melancolia como "estado de depressão mental, em que o sofrimento não é razoável...” (p. 151).

Em todas as três citações, o termo "depressão" refere-se a um sintoma. Kraepelin (1921), no entanto, usou "estados depressivos" como uma categoria genérica sob a qual ele incluiu a melancolia simplex, o estupor, a melancolia gravis, a melancolia fantástica e a melancolia delirante. No Reino Unido, esse grupo de distúrbios continuou a ser considerado como "melancolia". Assim, na famosa Nomenclatura de doenças elaborada por um comitê misto nomeado pelo Royal College of Physicians of London (1906), a melancolia foi classificada como uma "doença do sistema nervoso" (código 146), exibindo estados agudos, recorrentes ou crônicos. O Comitê informou que "a variedade quando conhecida deve ser classificada de acordo com as seguintes categorias: agitada, estupor, hipocondríaca, puerperal, do climatério, senil e de doença aguda ou crônica, ou devida a lesões" (p. 37). 


\section{Depressão endógena e melancolia}

O qualificador "endógeno", introduzido na virada do século, não tem sido particularmente esclarecedor (Masi, 1981). Conceitualmente baseado na velha "teoria da degeneração", ele carregou um contrabando semântico que não pode ser acomodado no século XX. Kraepelin propôs uma divisão das doenças mentais em exógenas e endógenas e reconheceu sua dívida para com Möbius (1893). A palavra "endógeno" (originalmente cunhada no contexto da classificação botânica por De Candolle em 1813; Heron, 1965) foi usada na psiquiatria fin de siècle como um termo "técnico" para se referir aos estados psicopatológicos que se acreditava resultarem da "degeneração" da semente humana (por exemplo, a histeria e a doença maníaco-depressiva) (Lewis, 1971). O restante dos distúrbios psiquiátricos eram "exógenos". A linha divisória não estava traçada entre o meio ambiente e o corpo (como é muitas vezes assumido) (Berrios, 1987), mas entre a doença decorrente da Anlage (conceito abstrato, que incluía tanto a genética quanto a psicogenicidade) e o restante das possíveis causas (Schiller, 1982).

\section{Os estados combinados}

Os historiadores clínicos não podem deixar de "garimpar" as referências que provem que no passado remoto alguém "descobriu" a doença maníaco-depressiva, e a Areteus, o capadócio, tem sido concedida a honra duvidosa (Kotsopoulos, 1986; Jellife, 1931). Estes esforços são de pouca utilidade, já que o conceito de doença que tornou possível o conceito de transtorno bipolar só se desenvolveu durante o século XIX.

As condições conceituais necessárias para a noção de insanidade alternada emergir foram: 1. A consolidação da visão anatomoclínica da doença; 2 . uma definição longitudinal de doença em oposição a uma transversal (a introdução deste princípio devemos a Kahlbaum e não a Kraepelin) (Berrios \& Hauser, 1988); 3. estabilidade na semiologia da afetividade, que inclui codificação de características tais como regularidade, intensidade, congruência, ritmo etc. (Berrios, 1985a); 4. disponibilidade de um conceito de personalidade, e 5. estratégias para diagnósticos diferenciais (por exemplo, per genus et differentiae).

Todas as cinco condições foram atendidas depois de 1850. Quase imediatamente após as insanidades circulares serem descritas, Falret (1854) e Baillarger (1854) embarcaram em uma disputa prolongada sobre a prioridade mas, com o benefício da retrospectiva, parece claro que ambos descreveram padrões 


\begin{tabular}{c|c} 
HISTÓRIA DA \\
PSICOPATOLOGIA, \\
POR GERMAN E. \\
BERRIOS \\
\hline
\end{tabular}

evolutivos incomuns (e rígidos) para a doença. Falret insistiu que a mania e a melancolia se alternariam em intervalos regulares; e Baillarger que entre as duas haveria um terceiro período (intercalado) de lucidez.

A psiquiatria europeia logo assumiu a condição. Em 1880 a Academia Francesa de Medicina abriu um concurso sobre ela (ganho por A. Ritti), e no mesmo ano o Comitê Inglês, responsável pela organização do Encontro de Psiquiatria de Londres de 1881, incluiu uma sessão para discutir a relevância clínica da nova doença.

Para se tornar mais aceitável e clinicamente útil, a doença precisava escapar das rígidas alternâncias sugeridas tanto por Falret quanto por Baillarger. Isto foi conseguido durante a década de 1880 , quando o conceito de "personalidade" alternante (uma forma atenuada da doença) tornou-se plenamente reconhecido, assim como sua base genética. Vários padrões e combinações foram descritos com relação à intensidade, duração e alternância dos três estados básicos (mania, melancolia e lucidez).

Houve, no entanto, algum debate sobre se a combinação seria uma coincidência e, afinal, não existiria a doença real alternada (Morel e Dagonet), ou se, se existisse, a apresentação isolada de mania ou depressão seriam, de fato, formes frustres da doença total (Marce, Foville, Ritti Luys, Billod, Meyer, Kirn, Krafft-Ebing e Karrer). Na verdade, foram feitos esforços para identificar as características clínicas que pudessem permitir o diagnóstico no exame transversal, eliminando assim a necessidade de observação longitudinal.

O diagnóstico diferencial foi feito em relação à mania pura, lipemania (depressão) e do "estado exaltado" da paralisia geral do insano. Não há espaço neste curto artigo para narrar este debate em detalhe. Uma importante questão clínica foi, por exemplo, a observação de que enquanto a mania pura era mais frequentemente caracterizada por alterações de pensamento e delírios, a mania (como parte de um estado combinado) apresentava mais frequentemente hiperatividade e elação (delírios de afeto, movimento e ação, como os franceses a chamavam) (Foville, 1882). Esta interessante hipótese clínica não foi totalmente testada.

\section{As consequências}

Kraepelin (1921) resolveu o problema do diagnóstico diferencial alegando que não havia diferenças reais entre esses estados: "a insanidade maníaco-depressiva, tal como será descrita nesta seção, inclui por um lado todo o domínio da assim chamada insanidade periódica e circular, por outro lado a mania simples, a maior 
parte dos estados mórbidos denominados melancolia e também um número não negligenciável de casos de amência" (p. 2). Se esta visão unitária é correta, ainda continua a ser considerado, mas aqueles que, até hoje, defendem a integração kraepeliniana devem lembrar que a "amência" (um conceito descrito por Meynert, 1890) não deveria ser esquecida em silêncio simplesmente porque pode revelar-se um embaraço clínico. A análise histórica mostra que Kraepelin poderia, afinal, ter tido razão em sua inclusão, já que a amência também pode se referir a estados de pseudodemência depressiva e maníaca (Berrios, 1985b; Bulbena \& Berrios, 1986). A integração de Kraepelin foi contestada por grandes figuras da psiquiatria europeia que queriam manter as melancolias (depressões unipolares) em um grupo separado. Um representante típico deste grupo foi Chaslin (1912).

\section{Conclusões}

Este breve artigo lidou com a história conceitual e o pano de fundo ideológico que tornou possível a transformação da noção pré-século XIX de melancolia nos conceitos de depressão e transtorno bipolar, a partir das mudanças médicas e psicológicas que operaram no início do século XIX até o trabalho integrativo de Kraepelin. A antiga noção de melancolia foi remodelada em significado e sua transição para a doença depressiva foi facilitada pelo conceito de lipemania de Esquirol, que, pela primeira vez, enfatizou a natureza afetiva primária da doença. Finalmente, uma vez que as condições conceituais tinham sido obtidas, a melancolia e a mania foram combinadas no conceito de insanidade alternante, periódica, circular, ou de forma dupla, os rígidos padrões descritivos anteriores da doença foram tornados flexíveis, este processo culminando com a sinopse final de Kraepelin.

O século XIX construiu seis princípios teóricos para o nosso atual conceito de doença maníaco-depressiva: era um transtorno "primário" do afeto, e não do intelecto ou cognição (Bolton, 1908), apresentava psicopatologia estável (Foville, 1882), tinha representação cerebral (Ritti, 1876); era periódico em sua natureza (Falret, 1854; Baillarger, 1854), era de origem genética (Foville, 1882), e tendia a aparecer em indivíduos com reconhecível predisposição de personalidade (Ritti, 1876). As causas reais dos episódios permaneceram de natureza endógena (Chaslin, 1912).

Todas estas características foram extraídas a partir da observação clínica, estatística descritiva e raciocínio lógico, e foram baseadas em suposições do século XIX a respeito de como a mente normal e patológica funcionariam. Que nós ainda compartilhamos destes pressupostos é melhor ilustrado por ainda 


\begin{tabular}{c|c} 
HISTÓRIA DA \\
PSICOPATOLOGIA, \\
POR GERMAN E. \\
BERRIOS \\
\hline
\end{tabular}

permanecermos presos naquilo que Kraepelin chamava de "círculo" da insanidade maníaco-depressiva.

\section{Referências}

Ackerknecht, E. Medicine at the Paris Hospital 1794-1848. Baltimore: Johns Hopkins Press, 1967.

ARnold, T. Observations on the Nature, Kinds. Causes and Prevention of Insanity, Leicester: G. Ireland, 1782. V. I.

Baillarger, J.G.F. Essai sur une classification des differents genres de folie. Annales Medico-Psychologiques, n. 5, p. 545-66, 1853.

Baillarger, J.G.F. De la folie à double-forme. Annales Médico-Psychologiques, n. 6, p. 367-91, 1854.

Baldwin, J.M. Dictionary of Philosophy and Psychology. London: MacMillan, 1901.

Battie, W. A Treatise on Madness. London: J. Whiston and B. White, 1758.

BeER, D.; Berrios, G. E. (1988). The notion of Unitary psychosis: a conceptual history. History of Psychiatry, n. 5, p. 13-6, 1994.

BÉGIN, J. (1834) Nostalgie. In: Dictionnaire de Médicine et de Chirurgie Pratiques. Paris: Mequignon Marvis, 1834. V. 12, p. 76-84.

Berrios, G. E. Delirium and confusion in the 19th century: a conceptual history. British Journal of Psychiatry, n. 139, p. 439-49, 1981a.

Berrios, G. E. Stupor: a conceptual history. Psychological Medicine, n. 11, p. 677-688, 1981b.

Berrios, G. E. Descriptive psychopathology: conceptual and historical aspects. Psychological Medicine, n. 14, p. 303-13, 1984.

Berrios, G. E. The psychopathology of affectivity: conceptual and historical aspects. Psychological Medicine, n. 15, p. 745-58, 1985a.

Berrios, G. E. 'Depressive pseudodementia' or 'melancholic dementia': a 19th century view. Journal of Neurology, Neurosurgery and Psychiatry, n. 48, p. 393-400, 1985 b.

Berrios, G. E. Historical aspects of the psychoses: 19th century issues. British Medical Bulletin, n. 43, p. 484-98, 1987.

Berrios, G. E. Depressive and manic states during the 19th century. In: Gorgotas, D.; Cancro, T. (eds.). Handbook of Affective Disorders. New York: Elsevier Science Publishers, 1988a. Cap. 2, p. 13-25. 
BerRios, G. E. The historical development of abnormal psychology. In: MiLLER, E.; CoOPER, P. J. Textbook of Abnormal Psychology. London: Churchill Livingstone, 1988b.

Berrios, G.E.; Hauser, R. The early development of Kraepelin's ideas on classification: a conceptual history. Psychological Medicine, v. 18, n. 4, p. 813-21, 1988.

Billod, E. Des diverses formes fr lypémanie. Annales Médico-Psychologiques, n. 2, p. 308-38, 1856.

Botton, J. S. Maniacal-depressive insanity. Brain, n. 31, p. 301-18, 1908.

Bowman, I. A. William Cullen (1710-1790) and the primacy of the nervous system. PhD Thesis, Indiana University, 1975.

Briand, M.; Azemar, C. La 'folie maniaque depressive'au XVllle siècle. Annales Médico-Psychologique, n. 12, p. 18-24, 1923.

Bucknill, J.C.; Tuke, D.H. A Manual of Psychological Medicine. London: Churchill, 1858.

Bulbena, A.; Berrios, G.E. Pseudodementia: facts and figures. British Journal of Psychiatry, n. 148, p. 87-93, 1986.

Burton, R. The Anatomy of Melancholy. London: Chatto and Windus, 1883.

Chaslin, P. Eléments de sémiologie et clinique mentales. Paris: Asselin et Houzeau, 1912.

CRICHTON, A. An Inquiry into the Nature and Origin of Mental Derangement. London: Cadell, Junior, and Davies, 1798.

DAnion, J.M.; KePPI, J.; Singer, L. Une approche historique de la doctrine des dégénérescences et des constitutions psychopatiques. Annales Médico-Psychologiques, n. 146, p. 271-80, 1985.

Delasiauve, L.J.F. Du diagnostic différentiel de la lypémanie. Annales MédicoPsychologiques, n. 3, p. 380-442, 1856.

Del Pistoia, L. Le probléme de la temporalite dans la psychiatrie française classique. L'Evolution Psychiatrique, n. 36, p. 445-74, 1971.

Desruelles, M.; Léculier, P.; Gardien, M. P. Contribution a l'histoire des classifications psychiatriques. Annales Médico-Psychologiques, n. 92, p. 637-75, 1934.

DowbigGin, I. Degeneration and hereditarianism in French mental medicine 1840-1890. In: Bynum, W.F.; Porter, R.; ShePherd, M. (eds.). The Anatomy of Madness. London: Tavistock, 1985. v. I, p. 189-232.

DrABKIN, I.E. Remarks on ancient psychopathology. Isis, n. 46, p. 223-234, 1955. 


\begin{tabular}{c|c} 
HISTÓRIA DA \\
PSICOPATOLOGIA, \\
POR GERMAN E. \\
BERIOS \\
\hline
\end{tabular}

Esquirol, J.E. Mélancolie. In: Dictionnaire des Sciences Médicales par une Société de Médicins et de Chirurgienms. Paris: Panckoucke, 1820.

FALRet, J.P. Mémoire sur la folie circulaire. Bulletin de l'Académie de Médicine, n. 19, p. 382-415, 1854.

Feuchtersleben, E. von The Principles of Medical Psychology. London: Sydenham Society, 1847.

Flashar, H. Melancholie und Melancholiker in den Medizinischen Theorien der Antike. Berlin: W. D. Gruyter, 1966.

Foville, A. Nomenclature et classification des maladies mentales. Annales MédicoPsychologiques, n. 30, p. 5-35, 1872.

FovilLe, A. Folie à double-forme. Brain, n. 5, p. 288-323, 1882.

FriedLander, R. Benedict Augustin Morel and the development of the theory of dégénérescence. $\mathrm{PhD}$ thesis. University of California, 1973.

Griesinger, W. Mental Pathology and Therapeutics. Trad. C. L. Robertson; J. Rutherford. London: New Syndenham Society, 1867.

Gull, W. W. A Collection of the Published Writing of W.W. Gull (ed. T. D. Acland). London: The New Syndenham Society, 1894.

Haslam, J. Observations on Madness and Melancholy. 2. ed. London: Callow, 1809.

Heiberg, J.L. Geisteskrankheiten in klassischen Altertum. Zeitschrift für Psychiatrie, n. 86, p. 1-44, 1927.

Heron, M.J. A note on the concept endogenous-exogenous. British Journal of Medical Psychology, n. 38, p. 241-45, 1965.

HoeldtKe, R. The history of associationism and British medical psychology. Medical History, n. 11, p. 46-64, 1967.

Huber, J.P. Aretée de Cappadoce et la psychose maniaco-dépressive. L'Information Psychiatrique, n. 61, p. 1375-86, 1985.

JACKSON, S.W. Galen on mental disorder. Journal of the History of the Behavioural Sciences, n. 5, p. 365-84, 1969.

JACKSON, S.W. Two sufferers' perspectives on melancholia: 1690-1790. In: WALLACE, E.R. IV; Pressly, L.C. (eds.). Essays in the History of Psychiatry. Columbia: R. L. Bryan, 1981.

JACKSON, S.W. Melancholia and mechanical explanation in eighteenth century medicine. Journal of the History of Medicine, n. 38, p. 298-319, 1983.

JACKSON, S.W. Melancholia and Depression. New Haven: Yale University Press, 1986. 
$\begin{array}{lllllll}R & E & V & \text { I } & S & T & A\end{array}$

LATINOAMERICANA

DE PSICOPATOLOGIA

F U N D A M E T A L

JACQUART, D. La rèflexion médicale médiévale et l'apport arabe. In: Postel, J.; QueTEL, C. (eds.). Nouvelle Histoire de la Psychiatrie. Paris: Privat, 1983. p. 43-53.

JELLIFFE, S.E. Some historical phases of the manic-depressive synthesis. In: Association for Research in Nervous and Mental Disease. Baltimore: William \& Wilkins, 1931. v. XI, p. 3-47.

Jones, W.H.S. Hippocrates General Introduction. Trad. inglesa. London: Heinemann Ltd., 1972. v. 1.

Kotsopoulos, S. Aretaeus the Cappadocian on mental illness. Comprehensive Psychiatry, n. 27, p. 171-79, 1986.

Kraepelin, E. Manic-depressive Insanity and Paranoia. Trad. R. M. Barclay da 8. ed. Edinburgh: Livingstone, 1921.

Kroll, J.; Bachrach, B. Sin and mental illness in the Middle Ages. Psychological Medicine, n. 14, p. 507-14, 1984.

Laín Entralgo, P. Historia de la Medicine. Barcelona: Salvat, 1978.

LANTÉRI LAURA, G. La chronicité dans la psychiatrie modern française. Annales ( $\mathrm{Pa}$ ris), n. 3, p. 548-68, 1972.

LaRson, J. L. Reason and Experience. The Representation of Natural Order in the Work of Carl Von Linné. Berkeley: University of California Press, 1971.

Leibbrand, W.; Wettley, A. Der Wahnsinn. Geschichte der abendländischen Psychopathologie. Freiburg: Karl Alber, 1961.

LEwIs, A. Melancholia: A historical review. Journal of Mental Science, n. 80, p. 1-42, 1934.

Lewis, A. 'Endogenous' and 'exogenous': a useful dichotomy? Psychological Medicine, n. 1, p. 191-96, 1971.

Llopis, B. La psicosis única. Archivo de Neurobiología, n. 17, p. 3-39, 1954.

LoóPEZ PiñERo, J.M. Historical Origins of the Concept of Neuroses. Trad. D. Berrios. Cambridge: Cambridge University Press, 1983.

MADDEN, J.S. Melancholy in medicine and literature: some historical considerations. British Journal of Medical Psychology, n. 39, p. 125-30, 1966.

MAsi, C. Histoire des psychoses endogènes. L'Information Psychiatrique, n. 57, p. 57-72, 1981.

Mayne, R.G. An Expository Lexicon of the Terms, Ancient and Modern, in Medical and General Science. London: Churchill, 1860.

Menninger, K.; Ellenberger, H.; Pruyser, P.; Mayman, M. The unitary concept of mental illness. Bulletin of the Menninger Clinic, n. 22, p. 4-12, 1958. 


\begin{tabular}{c|c} 
HISTÓRIA DA \\
PSICOPATOLOGIA, \\
POR GERMAN E. \\
BERIOS \\
\hline
\end{tabular}

Meynert, T. Klinische Vorlesungen über Psychiatrie auf Wissenschaftlichen Grundlagen für Studierende und Arzte, Juristen und Psychologen. Vienna: Braumüller, 1890.

Möвıus, P.J. Abriss der Lehre von den Nervenkrankheiten. Leipzig: Abel, 1893.

Moreau de Tours, J. La Psychologie Morbide. Paris: Victor Masson, 1859.

Morel, B.A. Traité des Dégénérescences Physiques, Intellectuelles et Morales de l'Espèce Humaine. Paris: Baillière, 1857.

Pinel, P. Traité Médico-Philosophique sur l'Aliénation Mentale. 2. ed. Paris: Brosson, 1809.

Prichard, J.C. A Treatise on Insanity and Other Disorders Affecting the Mind. London: Sherwood, Gilbert and Piper, 1835.

Rauchs, P. La 'nostalgie' chez Philippe Pinel. Un nouvel exemple de la 'distriaction' du maître de la Salpétrière. L'Evolution Psychiatrique, n. 50, p. 759-63, 1985.

RéGis, E. Manuel Pratique de Médicine Mentale. Paris: Octave Doin, 1885.

RitTı, A. Folie à double-forme. In: Dictionnaire Encyclopedique des Sciences Medicales. (ed. A. Dechambre). Paris: Aselin, 1976. V. 3, 4ª serie, p. 321-39.

RENNERT, H. Wilhelm Griesinger und die Einheitpsychose. Wissenschaftliche Zeitschrift der Humbolt-Universitat, n. 17, p. 15-6, 1968.

Roccatagliata, G. Storia della Psichiatria Antica. Milano: Hoepli, 1973.

Rosen, G. Nostalgia: a ‘forgotten’ psychological disorder. Psychological Medicine, n. 5, p. 340-54, 1975.

Royal College of Physicians of London. The Nomenclature of Diseases. 4. ed., 3. rev. London: HMSO, 1906.

Rush, B. Medical Inquiries and Observations upon the Diseases of the Mind. Philadelphia: Kimber \& Richardson, 1812.

Savage, G.H. Insanity and Allied Neuroses, Practical and Clinical. London: Cassell and Company, 1898.

SchiLler, F. A Möbius Strip. Berkeley: University of California Press, 1982.

SEDLER, M.J.; Dessain, E.C. Falret's discovery: the origin of the concept of bipolar affective illness. American Journal of Psychiatry, n. 140, p. 1127-33, 1983.

SiEgel, R.E. Galen on Psychology, Psychopathology and Function and Diseases of the Nervous System. Basel: Karger, 1973.

Simon, B. Mind and Madness in Ancient Greece. The Classical Roots of Modern Psychiatry. Ithaca: Cornell University Press, 1978. 
StARobinski, J. Historia del tratamiento de la melancolia desde los origenes hasta 1900. Acta Psychosomática, n. 3. Geigy S.A. Basilea, 1962.

Tracy, T. Physiological Theory and the Doctrine of the Mean in Plato and Aristotle. The Hague: Mouton, 1969.

VIÉ, M.J. Sur l'existence d'entités morbides en psychiatrie, l'utilité et l'orientation de l'effort nosologique. Annales Médico-Psychologiques, n. 98, p. 347-58, 1940.

Vliegen, J. Die Einheitpsychose. Stuttgart: Enke, 1980.

WaLSER, H.H. Melancholie. Medizingeschichtlicher Sichtung und Therapie, Umschau, n. 25, p. 17-21, 1968.

WALSER, R.D. What became of the degenerate. Journal of the History of Medicine and Allied Sciences, n. 11, p. 422-29, 1956.

Warren, H.C. History of the Association Psychology. New York: Scribners and Sons, 1921.

Wettley, A. Zur Problemgeschichte der 'dégénérescences'. Sudhoffs Archiv, n. 43, p. 193-212, 1959.

\section{Resumos}

(Melancholia and Depression during the 19th Century: A Conceptual History)

This article historically examines the ideological background that opened the way to the transformation of the notion of melancholia into the concepts of depression and bipolar disorder, based on changes in medicine and psychology during the 19th century. The older notion of melancholia was remodeled and its transition to the concept of depression disorder was facilitated by the concept of lipemania, introduced by Esquirol, who first emphasized the primarily affective nature of the disease. Finally, once the necessary conceptual conditions had been attained, melancholia and mania were merged into the concept of periodic, circular and alternating (or even simultaneous) insanity. Consequently, its strict descriptive standards were relaxed, culminating in Kraepelin's synopsis.

Key words: Melancholia, bipolar disorder-history, depressive disorder-history, history of psychiatry

(Mélancolie et dépression au cours du XIX siècle: une histoire conceptuelle)

Cet article examine du point de vue historique le contexte idéologique qui a permit la transformation de la notion de mélancolie en concepts de dépression et de trouble bipolaire à partir de l'évolution médicale et psychologique qui a eu lieu au cours 
HISTÓRIA DA

PSICOPATOLOGIA,

POR GERMAN E.

BERRIOS

du XIX siècle. L'ancienne notion de mélancolie est révisée et sa transformation en notion de maladie dépressive s'opère entre autre grâce au concept de lypémanie d'Esquirol qui a été le premier à souligner le caractère affectif primaire de la maladie. Les conditions conceptuelles nécessaires établies, la mélancolie et la manie sont transformées en concept de la folie alternante, périodique, circulaire, ou double. Ses normes descriptives rigides sont assouplies, ce qui finalement abouti à la synthèse de Kraepelin.

Mots clés: mélancolie, histoire du trouble bipolaire, histoire du trouble dépressif, histoire de la psychiatrie

(Melancolía y depresión durante el siglo XIX: uma historia conceptual)

Este artículo analiza históricamente el background ideológico que hizo posible la transformación de la noción de melancolía en los conceptos de depresión y trastorno bipolar, a partir de los cambios médicos y psicológicos ocurridos en el decorrer del siglo XIX. La noción antigua de melancolía fue remodelada y su transición para la enfermedad depresiva fue facilitada por el concepto de lipemania de Esquirol, quien, por primera vez, enfatizó la naturaleza afectiva primaria de la enfermedad. Finalmente, obtenidas las condiciones conceptuales necesarias, la melancolía y la manía fueron combinadas en el concepto de insanidad alternante, periódica, circular, o de forma dupla, sus patrones rígidos descriptivos fueron flexibilizados, culminando este proceso en la sinopsis de Kraepelin.

Palabras clave: Melancolía, trastorno bipolar-historia, trastorno depresivo-historia, historia de la psiquiatría

(Melancholie und Depression im 19. Jahrhundert: eine konzeptionelle Geschichte)

In diesem Beitrag wird der ideologische Hintergrund untersucht, der die Verwandlung der Auffassung von Melancholie in den Begriffen der Depression und der Bipolarität ermöglicht hat. Ausgangspunkte sind ärztliche und psychologische Veränderungen im 19. Jahrhundert. Die frühere Auffassung von Melancholie wurde umformuliert und der Übergang ihrer Anerkennung als depressive Krankheit wurde durch das Monomanie Konzept von Esquirol erleichtert, der zum ersten Mal die primär affektive Natur der Krankheit hervorhob. Und schließlich, aufgrund der erlangten notwendigen Begrifflichkeit, wurden Melancholie und Manie (=Wahn) zusammengeführt und haben den Begriff des abwechselnden, periodischen Wahn-sinns, der im Kreis oder als Doppelerscheinung läuft gebildet. Seine strengen deskriptiven Standards wurden flexibilisiert und dieser Prozess erfuhr seinen Höhepunkt in der Zusammenfassung von Kraepelin.

Schlüsselwörter: Melancholie, Geschichte der bipolaren Störung, Geschichte der depressiven Störung, Geschichte der Psychiatrie 


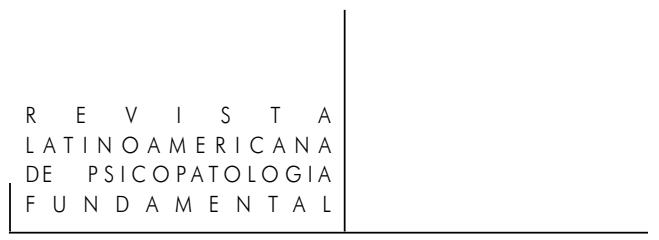

Citação/Citation: BerRIos, G.E. Melancolia e depressão durante o século XIX: uma história conceitual. Revista Latinoamericana de Psicopatologia Fundamental, São Paulo, v. 15, n. 3, p. 590-608, setembro 2012.

Editor do artigo/Editor: Prof. Dr. Manoel Tosta Berlinck

Recebido/Received: 28.9.2011 / 9.28.2011 Aceito/Accepted: 3.11.2011 /11.3.2011

Copyright: () 2009 Associação Universitária de Pesquisa em Psicopatologia Fundamental/ University Association for Research in Fundamental Psychopathology. Este é um artigo de livre acesso, que permite uso irrestrito, distribuição e reprodução em qualquer meio, desde que o autor e a fonte sejam citados / This is an open-access article, which permits unrestricted use, distribution, and reproduction in any medium, provided the original author and source are credited.

Financiamento/Funding: $\mathrm{O}$ autor declara não ter sido financiado ou apoiado / The author has no support or funding to report.

Conflito de interesses/Conflict of interest: $O$ autor declara que não há conflito de interesses / The author declares that has no conflict of interest.

\section{German E. Berrios}

Médico e filósofo pela Universidad Nacional de San Marcos, Lima, Peru; Psiquiatra; Neurologista; Psicólogo; Filósofo; Historiador e Filósofo da ciência (Oxford University, England); Professor de Neuropsiquiatria e de Epistemologia da Psiquiatria (University of Cambridge, England), desde 1976; Neuropsiquiatra e Chefe do Departamento de Neuropsiquiatria do Hospital Addenbrooke, University of Cambridge, por 32 anos; Coordenador do Comitê de Ética em Pesquisa com Humanos na mesma universidade, por vinte anos; Editor Responsável de History of Psychiatry; Autor de 14 livros, incluindo The History of Mental Symptoms, Descriptive Psychopathology since 19th Century (Prêmio Nacional BMA, 1997), A History of Clinical Psychiatry (com Roy Porter), e Delirio (com F. Fuentenebro) e mais de 400 artigos e capítulos de livros; Membro do Royal College of Psychiatrists; da Associação Britânica de Psicologia e da Academia Britânica de Ciências Médicas; Membro Vitalício do Robinson College, Cambridge; doutor Honoris Causa da Universidade de Heidelberg (Alemanha), da Universidade Nacional Mayor de San Marcos (Peru) e da Universidad Autónoma de Barcelona (Espanha); Grão Oficial da Ordem del Sol (Condecoração do Governo Peruano, 2007); prêmio Ramon y Cajal 2008 concedido pela Asociación Internacional de Neuropsiquiatria.

University of Cambridge

Box 189, Hills Road

Cambridge,

UK CB2 2QQ

e-mail: geb11@cam.ac.uk 